\title{
Face Recognition System based on SURF and LDA Technique
}

\author{
Narpat A. Singh \\ Department of CSE, CT Institute of Engineering Mgt \& Technology, Punjab, India \\ E-mail: narpatsingh23@gmail.com \\ Manoj B. Kumar \\ Department of ECE, CT Institute of Engineering Mgt. \& Technology, Punjab, India \\ E-mail: drmanojkumarindia@gmail.com \\ Manju C. Bala \\ Department of CSE, CT Institute of Engineering Mgt. \& Technology, Punjab, India \\ E-mail: manju.ctgroup@gmail.com
}

\begin{abstract}
In the past decade, Improve the quality in face recognition system is a challenge. It is a challenging problem and widely studied in the different type of images to provide the best quality of faces in real life. These problems come due to illumination and pose effect due to light in gradient features. The improvement and optimization of human face recognition and detection is an important problem in the real life that can be handles to optimize the error rate, accuracy, peak signal to noise ratio, mean square error, and structural similarity Index. Now-a-days, there several methods are proposed to recognition face in different problem to optimize above parameters. There occur many invariant changes in human faces due to the illumination and pose variations. In this paper we proposed a novel method in face recognition to improve the quality parameters using speed up robust feature and linear discriminant analysis for optimize result. SURF is used for feature matching. In this paper, we use linear discriminant analysis for the edge dimensions reduction to live faces from our data-sets. The proposed method shows the better result as compare to the previous result on the basis of comparative analysis because our method show the better quality and better results in live images of face.
\end{abstract}

Index Terms-Image processing, Feature Extraction, Detection, Binarization, Discrete wavelet transform, Gabor filter, Speed up robust features, Linear Discriminant Analysis.

\section{INTRODUCTION}

Face recognition system is a part of biometrics. Face recognition system is mainly created for security purpose in real life. Humans are very good to check identity and verify to recognizing faces and complex patterns. Now-adays, a number of applications are used by the army or police forces and civilians need to effective face recognition in different ways. The main significant purpose of face recognition system is to identification, verification and physical access control. When an access point gets an image of anyone's face and compares it with the prestored database of faces. If the image is matched, access is granted for further processing and result action is performed. The areas where red alert or have a high level security, there should be different and specials security checks such as face and checking cards etc. This kind of face recognition system works in sensitive areas like air ports for facilitation of staff and other people to pass through different security levels without showing their identification. Applications where face verification can be used efficiently including secure transactions in business field such as e-commerce, m-commerce and banking. Face recognition can also be used in government security issues helps to provide national ID cards, passports, license, UID Aadhar Card etc. It can be helpful and successful in the checking for criminal records, enhancement of security by using surveillance cameras in conjunction using face recognition system, finding lost children's by using the images received from the cameras fitted at some public places, knowing in advance if some VIP is entering the hotel, Detection of a criminal at public place, it can be used in different areas of science and geographical area for comparing entity with a set of entities in huge data, etc.

\section{RELATED WORK}

Face recognition is used for both verification and identification in the real life. Detailed information of illumination and pose invariant face recognition techniques can found for feature extraction and feature selection for future work. Variations in images of live face due to illuminating, pose and lighting conditions should not better its efficiency. Aneesh M U et al. proposed an algorithm for the application to select the feature subset from the database is commonly known as feature selection and PSO algorithm is used as bird flocking and fish- 
schooling, this algorithm is also known as on binary PSO [1]. Matthew A. Turk et al. identify an approach to the detection and identification of human faces which tracks human faces of the individuals to face recognition as $2 \mathrm{D}$ recognition. The face images are projected on to the feature space (Eigen faces) images and find the variation among the images of face. The face space framework provides the ability to learn to recognize new faces as unsupervised manner to provide the solution of the problems through Eignfaces [2]. Priya Sisodia et al. worked the useful property of Gabor in the face datasets with robustness against slight object distortion, rotation and variation in illumination of due to light. In this paper they used a number of parameter use to represent Gabor feature and space complexity reduction with help of SVM classifier on facial data. Gabor filter removes variability that occurs due to changes in the lighting and noises [3]. Zhenhua Chai et al. gave a new method that allows for obtain more robust histograms of local patterns by using a more discriminative spatial division strategy is use the face measures. Spatial histograms are more suitable to obtain from regions clustered pixel relations by making better use of spatial information. PCA and whitening process are applied on the face image for the final vector dimension reduction to face recognition [4]. Jun Wang et al. is applied modifications of Hausdroff distance measurements by using k-nearest neighbors to obtain the average distance from each point in the model image with the test image KNNHD's various degree. The average of the distances to the k-nearest points is more suitable than the distance to one single point [5]. Chandrappa D N et al. are used techniques Gabor wavelets and morphological shared weighted neural network based automatic face recognition. These techniques used to transform images as independent of gray-level shifts. Face detection is performed under the MSWNN that recognize all the human faces in different environments for multi-view recognition [6]. Muhammad Sharif et al. proposed an Elastic Bunch Graph Map (EBGM) algorithm to implement face recognition system using by Gabor filters and calculate maximum intensity points in each filter image at different angles and orientation [7]. Dong Hui et al. applied matching SURF algorithm to detect the points and match points through high time efficient KD-tree nearest neighbor searching methods and find the results better as compare to SIFT matching algorithm and used the Haar wavelet to measure the points. SURF algorithm is used as the image's scale rotation [8]. Shih-wei Lin et al. proposed on linear discriminant analysis as classification method. LDA shows high correlation between features and noise in the images of the face. The PSO provides the better solution using LDA to determine best feature subset. The PCA based feature reused to increase the accuracy of the system. Data without feature selection may be redundant or noisy which may degrade classification accuracy rate [9], [11]. David Barina presented the use of twodimensional Gabor wavelets in image processing. The key idea of this work is to utilize a Gabor wavelet as a multi-scale partial differential operator of a given order. The best results are obtained with the derivative of the
Gaussian function which is closely followed by the Gabor wavelet using the discontinuous Haar wavelet [10].

\section{CATEgORIES OF FACE ReCOGNITION AND PROPOSED MODEL}

Holistic methods are methods which identify a face using the whole face images as input and extract the overall features. Feature based are methods which are used the local facial features for recognition (like eyes, mouths, fiducial points etc.). Hybrid methods are methods which are used both feature based and holistic features to recognize a face. In the proposed work we have used hybrid method is to improve the face quality parameters such as the error rate minimization, PSNR, MSE, Accuracy, and SSIM according to need of the secure whole world. The proposed system in figure 1, uses the own datasets for the live face images in the face recognition system. We have proposed discrete wavelet transform, Gabor filter and Gaussian filter, SURF and LDA. Discrete wavelet transform is used to feature detection and feature selection. It is apply by using two filter that is Gabor and Gaussian filter. SURF is used for feature point matching. If there is minimum distance between the face points then the system reduces the point distance and measures through Hausdroff classifier that improve the efficiency of the face recognition system. Gabor filter is used also for feature detection to provide the quality of face images. Gabor filter works on the different angle and rotation with orientation.

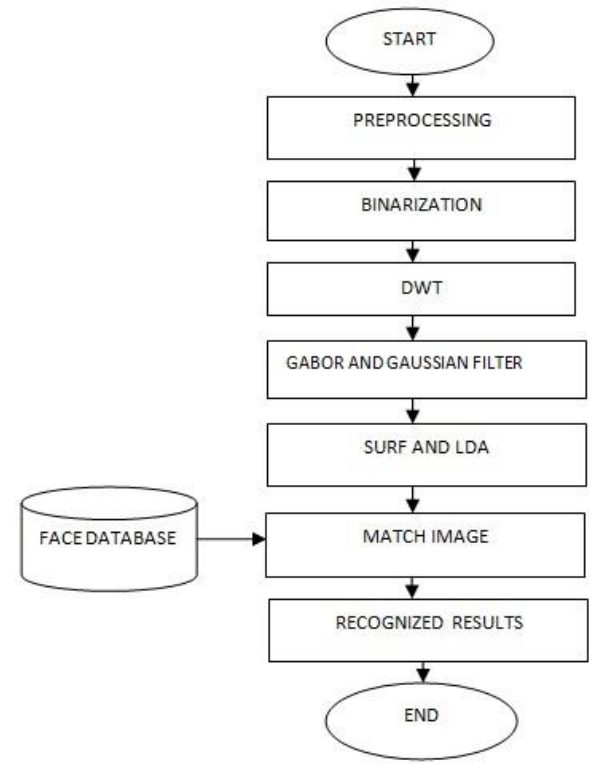

Fig.1. Proposed model of face recognition system

\section{Discrete WAVELET TRANSFORMS (DWT)}

An efficient implementation of DWT in jpeg2000 is designed with low memory and high pipeline architecture. Discrete wavelet transform is to reduce the computation 
time and resource requirement [19]. Wavelet transform has recently most popular for analysis, de-noising and compression of signals and images. Discrete wavelet transform (DWT) are applied to discrete data sets and produce discrete output, it is used to map data from the time domain to the wavelet domain. Wavelet domain defines the $n \times n$ dimensions of the matrices to inputs of size $n$. Depending on the boundary conditions; such matrices can be orthogonal or closed to the orthogonal. When the matrix is orthogonal it denotes to the rotation in the wavelet domain. DWT are used in two levels that is single level and multilevel, we have used the multilevel wavelet in our model $(\mathrm{cA}, \mathrm{cD}, \mathrm{cE}, \mathrm{cH}, \mathrm{cV})$ in which $\mathrm{cA}$ is used for approximation, $\mathrm{cD}$ for detail coefficients. DWT performs a multi-resolution analysis of a signal with localization in both time and frequency domain.

\section{GABOR FILTERS}

Gabor filter is used in many other areas such as finger print recognition, iris recognition, and character recognition for verification of signature in different languages. In image processing, a Gabor filter is a linear filter used for edge detection. It works into sub blocks. It is directly related to Gabor wavelets [12], [17], in this we can be design a number of dilations and rotations. It is band pass filters with tunable orientation and radial frequency bandwidths. Gabor filter is used for the quality purpose of the face images in our database. It helps to find the quality features in the human face images. If we study the wide details of Gabor filter it is best suited to find the whole information of the live human face image for the security purpose. In this paper we have used Gabor filter for more suitable quality and best feature extraction through different rotation of the image in whole face recognition.

A 2-D Gabor function is defined as in "(1)":

Complex form:

$g(x, y, \lambda, \theta, \chi, \sigma, \gamma)=\exp \left(-\frac{x^{\prime 2}+\gamma^{\prime 2} x^{\prime 2}}{2 \sigma^{2}}\right) \exp \left(i\left(2 \pi \frac{x^{\prime}}{\lambda}+\chi\right)\right)$

Real part:

$g(x, y, \lambda, \theta, \chi, \sigma, \gamma)=\exp \left(-\frac{x^{\prime 2}+{\gamma^{\prime}}^{2} x^{\prime 2}}{2 \sigma^{2}}\right) \cos \left(i\left(2 \pi \frac{x^{\prime}}{\lambda}+\chi\right)\right)$

Imaginary part:

$$
g(x, y, \lambda, \theta, \chi, \sigma, \gamma)=\exp \left(-\frac{x^{\prime 2}+\gamma^{\prime 2} x^{\prime 2}}{2 \sigma^{2}}\right) \sin \left(i\left(2 \pi \frac{x^{\prime}}{\lambda}+\chi\right)\right)
$$

Where

$$
x^{\prime}=x \cos \theta+y \sin \theta
$$

$$
y^{\prime}=-x \sin \theta+y \cos \theta
$$

The Gabor filters bank can be obtained by scale and rotation of $\mathrm{g}(\mathrm{x}, \mathrm{y})$, i.e;

$$
g_{m, k}(x, y)=a^{-m} g(x, y)
$$

In image processing, we have used the Gabor filter for the feature extraction.

Gabor function can be calculated for the linear filter:

$$
\text { Gabor function }=\text { Gaussian } * \text { Fourier }
$$

Gabor filter is a band pass filter for unidirectional signals in image processing. The role of Gabor filter to extracts the information from the noisy and blurs faces which are not seeing clear in the light and some angle. The works with filter bank and Gabor space. In filter banks, the Gabor filters with various scales and rotations with different angles. In this, $\lambda$ specifies the cosine factor of the Gabor function in "(1)" and $\theta$ specifies normal to parallel of the Gabor function and $\gamma$ specifies the elliptically of Gaussian factor. The value of orientation and wavelength may be change in the range from 2 to 256 according to the pixels of the image.

\section{SURF TECHNIQUE}

In this paper we have used the SURF technique to match the feature point between two images at the same time [8]. It is same as the SHIFT but the speed of the SURF is faster.

\section{LDA TECHNIQUE}

The basic idea of LDA is to find the linear transformation such as features clustered technique is used with scatter matrix analysis. The ratio of the determinant of the between class scatter matrix of the projected samples to the within class scatter matrix of the projected samples can be defined as following as in "(8)":

$$
A=\max \left[\frac{\text { Test number of samples }}{\text { Total number of samples }}\right]
$$

For the classification method, the linear discriminant functions are:

$$
\mathrm{D}_{\mathrm{i}}(\mathrm{X})=\mathrm{A}^{* \mathrm{~T}}\left(\mathrm{X}-\mu_{\mathrm{i}}\right), \mathrm{i}=1,2,3,4 \ldots \ldots, \mathrm{m}
$$

If we want to reduce the dimension of the matrix $n$ to $\mathrm{m}$, we can simply take $\mathrm{m}$ rows of matrix A as the transformation matrix that correspond to the eign values of $\mathrm{A}$. This can be happen when the number of training samples is smaller than the dimension of the samples vector [15]. LDA algorithm works for dual purpose such as dimensionality reduction and sub-space mapping. We can smoothly remove null space from the face images. 


\section{EXPERIMENT RESULT}

To find the result, we have calculated the results on the basis of parameters viz. error rate, psnr, mse, accuracy and ssim. First we have take the data set of 40 images of size of each image dimension $(150 \times 150)$ for live faces and this data set of 40 face images is categories into 4 parts. The first part contains 10 images of face, second part contains 20 images of face, third part contains 30 images of face, and last part contains 40 images of face.

\section{A. Error Rate}

To minimize the error rate in the face recognition system, we have to evaluate the following formula that is used in for better prediction of error rate using the formula as in "(10)":

$$
\% \text { Error }=\frac{\mid \text { Approximate value }- \text { Exact value } \mid}{\mid \text { Exact value } \mid} \times 100 \%
$$

Where ('|' symbols means absolute value due to negative becomes positive.)

Table 1. Comparison to error rate (\%)

\begin{tabular}{|c|c|c|c|c|}
\hline No. of images & 10 & 20 & 30 & 40 \\
\hline $\begin{array}{c}\text { Previous } \\
\text { Method }\end{array}$ & 14 & 14 & 14 & 14 \\
\hline $\begin{array}{c}\text { Proposed } \\
\text { Method }\end{array}$ & 7.7603 & 8.1122 & 9.8925 & 6.4987 \\
\hline
\end{tabular}

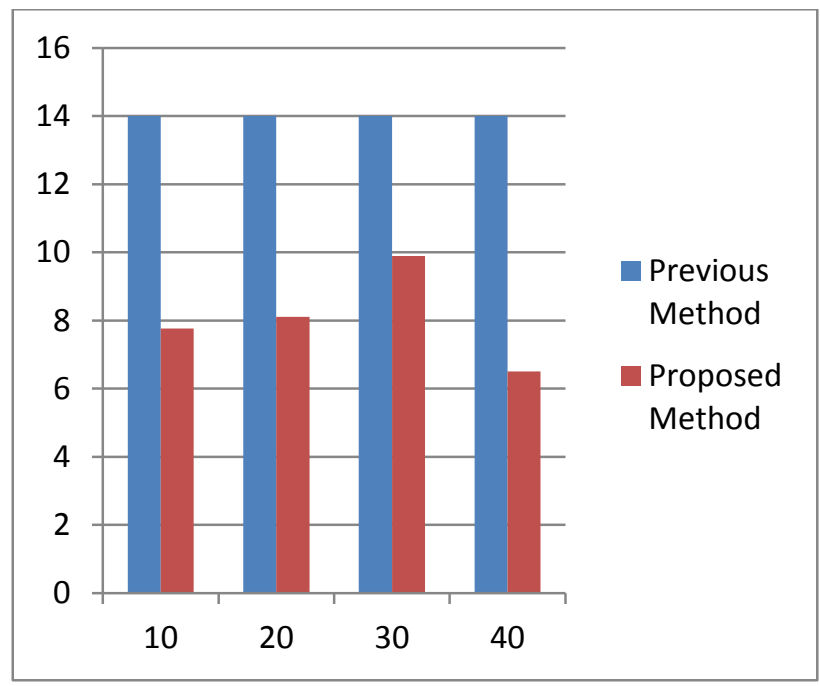

Fig.2. Comparison to Error Rate (\%)

In the Table 1, we have calculated the error rate in four parts and compared to proposed method and previous method. In previous work, five methods were used name as Eigenfaces, Laplacianfaces, Fisherfaces, FDS and Schurfaces, these method were used for minimize the error rate [11].In the previous methodology, Schurfaces got the minimum error rate $14 \%$, But in our methodology, we have used SURF and Linear Discriminant Analysis to find the results and find minimum error in all four parts.
By using LDA methodology, our results are better; in the "Fig. 2," we have compared the graph and map the values for the results in table1, in the previous method the error rate was $14 \%$ for the faces but we have calculated minimum error rate is $6.49 \%$ for the live faces. Our results are better in four cases from the previous method. For the data set of 40 images our error rate is very less as compare to the 10,20, and 30 images of face.

\section{B. Peak Signal to Noise Ratio}

The actual parameter computed is the peak signal-toreconstructed image measure which is called PSNR. Assume we are given a source image $I_{1}(m, n)$ and a processed image $\mathrm{I}_{2}(\mathrm{~m}, \mathrm{n})$ and computes the PSNR using the following as in "(11)":

$$
P S N R=10 \log _{10}\left(\frac{R^{2}}{M S E}\right)
$$

Table 2. Comparison to PSNR (db)

\begin{tabular}{|c|c|c|c|c|}
\hline $\begin{array}{c}\text { No. of imag- } \\
\text { es }\end{array}$ & 10 & 20 & 30 & 40 \\
\hline $\begin{array}{c}\text { Previous } \\
\text { Method }\end{array}$ & 28.6964 & 29.6655 & 30.9149 & 32.6758 \\
\hline $\begin{array}{c}\text { Proposed } \\
\text { Method }\end{array}$ & 34.1514 & 35.1205 & 36.3699 & 38.1308 \\
\hline
\end{tabular}

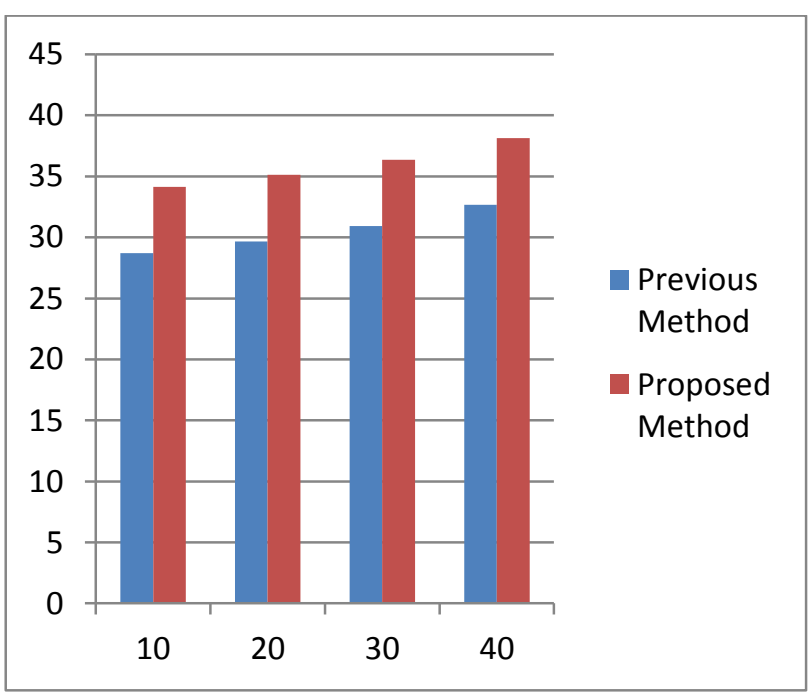

Fig.3. Comparison to PSNR (db)

In the Table 2, we have calculated the psnr. If psnr is high the image quality will also high. We have calculated the psnr value for the previous method and proposed method, in all cases our method shows the high psnr value in four parts and compared to proposed method and previous method. Our results are better in four cases from the previous method. For the data set of 40 images our psnr is very high as compare to the 10,20 , and 30 images of face. By the previous method the psnr highest value is $32.6758 \mathrm{db}$ but our proposed method gives the psnr value is 38.1308 that is very high. In the "Fig. 3," we have 
show the comparison to psnr of previous method and proposed method in form of graph.

\section{Mean Square Error}

The MSE represents the cumulative squared error between the original image and processed image. The lower the value of MSE, lower the error present in the image so better the quality of processed image.

$$
M S E=\sum_{\frac{1}{m n}}\left[I_{1}(m, n)-I_{2}(m, n)\right]^{2}
$$

$I_{1}(m, n)$ and $I_{2}(m, n)$ represents the original image and processed image respectively in "(12)". $\mathrm{M}$ and $\mathrm{N}$ are the numbers of rows and columns in the input image respectively.

Table 3. Comparison to MSE

\begin{tabular}{|c|c|c|c|c|}
\hline No. of images & 10 & 20 & 30 & 40 \\
\hline $\begin{array}{c}\text { Previous } \\
\text { Method }\end{array}$ & 61.4 & 56.4 & 51.4 & 46.4 \\
\hline $\begin{array}{c}\text { Proposed } \\
\text { Method }\end{array}$ & 25 & 20 & 15 & 10 \\
\hline
\end{tabular}

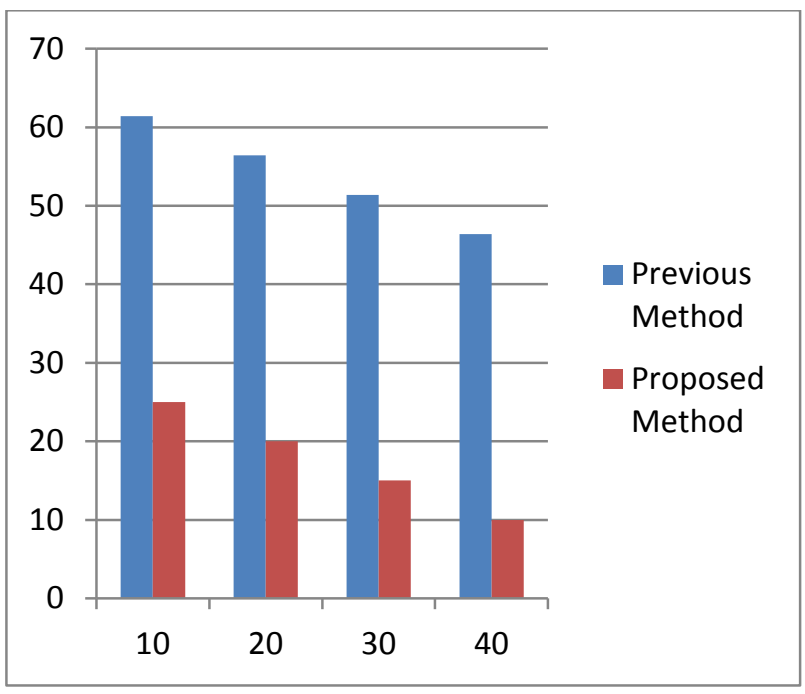

Fig.4. Comparison to MSE

In the Table 3, we have calculated the mse. If mse is low the image quality will high and if the mse is high then the image quality will be low. We have calculated the mse value for the previous method and proposed method, in all cases our method shows the low mse value in four parts and compared to proposed method and previous method. Our results are better in four cases from the previous method. For the data set of 40 images our psnr is very low as compare to the 10,20, and 30 images of face. By the previous method the mse lowest value is 46.4 but our proposed method gives the mse value is 10 that is very low. In the "Fig. 4," we have show the comparison to mse of previous method and proposed method in form of graph.

\section{Accuracy}

Accuracy is one of the most important issues in the face recognition system. For the better image quality, we have to need focus on the issues for the better calculation using the following formula as in "(13)":

$$
\text { Accuracy }=100-\left(\frac{\text { Difference }}{\text { Exact Value }} \times 100\right)
$$

Table 4. Comparison to accuracy (\%)

\begin{tabular}{|c|c|c|c|c|}
\hline No. of images & 10 & 20 & 30 & 40 \\
\hline $\begin{array}{c}\text { Previous } \\
\text { Method }\end{array}$ & 77.1098 & 76.9172 & 76.0555 & 77.8804 \\
\hline $\begin{array}{c}\text { Proposed Meth- } \\
\text { od }\end{array}$ & 79.2320 & 79.0394 & 78.1777 & 80.0026 \\
\hline
\end{tabular}

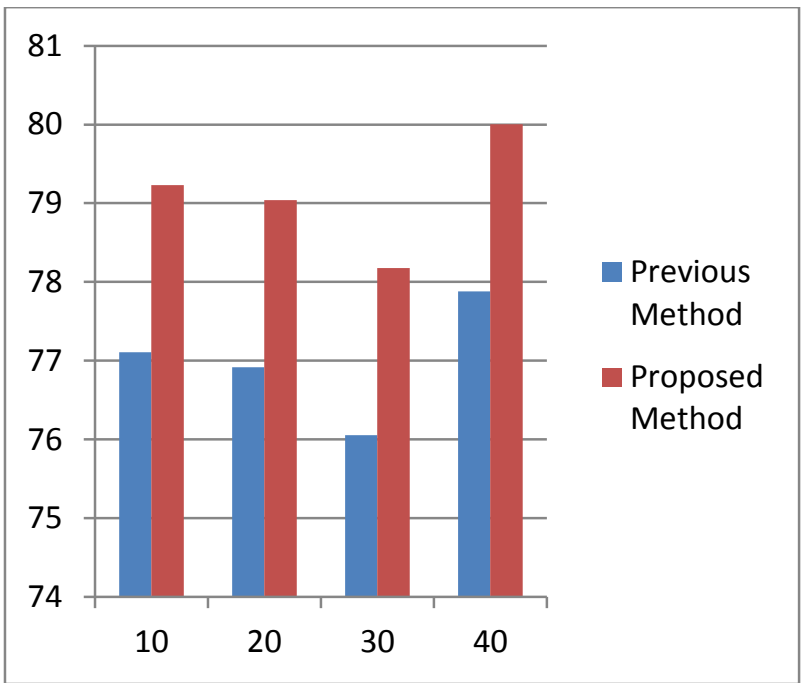

Fig.5. Comparison to Accuracy (\%)

In the Table 4, we have calculated the accuracy. If accuracy is high the image quality will high and if the accuracy is low then the image quality will be low. We have determined the accuracy value to improve face quality for the previous method and proposed method, in all cases our method shows the high accuracy value in four parts and compared to proposed method and previous method. Our results are better in four cases from the previous method. For the data set of 40 images our accuracy is very high as compare to the 10, 20, and 30 images of face. By the previous method the accuracy value is $77.8804 \%$ but our proposed method gives the accuracy value is $80.0026 \%$ that is very more. In the "Fig. 5," we have show the comparison to accuracy of previous method and proposed method in form of graph.

\section{E. Structural Similarity Index}

The Structural Similarity is used to determine improve the quality and performance of the peak-signal-to-noise ratio and mean square error. It is more suitable for the quality face information for the security purpose in our 
research work.

Table 5. Comparison to SSIM

\begin{tabular}{|c|c|c|c|c|}
\hline No. of images & 10 & 20 & 30 & 40 \\
\hline $\begin{array}{c}\text { Previous } \\
\text { Method }\end{array}$ & 0.7542 & 0.8789 & 0.7894 & 0.8779 \\
\hline $\begin{array}{c}\text { Proposed } \\
\text { Method }\end{array}$ & 0.9998 & 0.9999 & 1.000 & 1.000 \\
\hline
\end{tabular}

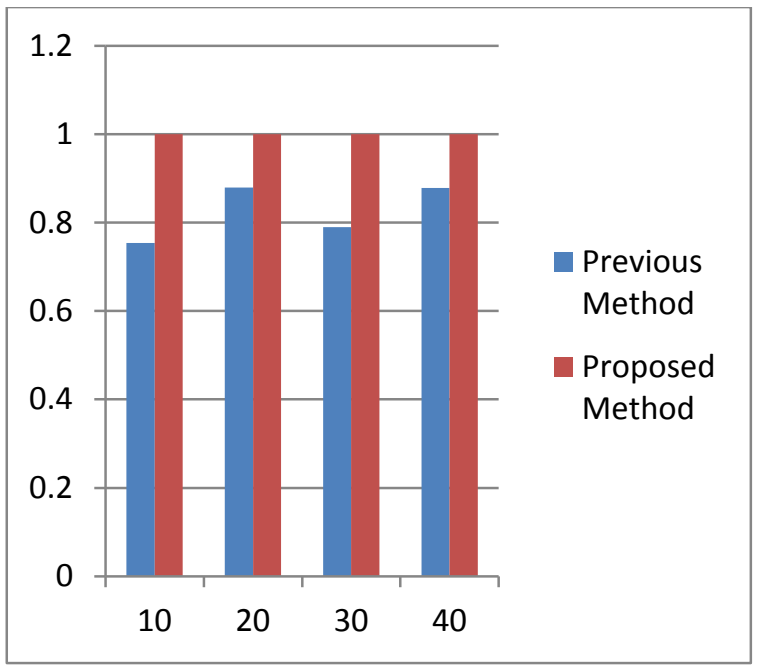

Fig.6. Comparison to SSIM

In the Table 5, we have calculated the SSIM. If SSIM is high the image quality will high. As the SSIM value will improve then the value of psnr and mse will also improved. We have calculated the SSIM value for the previous method and proposed method, in all cases our method shows the high SSIM value in four parts and compared to proposed method and previous method. Our results are better in four cases from the previous method. By the previous method the SSIM value remains within range 0.8779 to 0.8779 but our proposed method gives the SSIM value remains within range 0.9998 to 1.0000 . In the "Fig. 6," we have show the comparison to SSIM value of previous method and proposed method in form of graph.

\section{CONCLUSION AND FUTURE WORK}

The face recognition system is used widely in verification and identification for the security. Various image processing technique and methods are used to improve the face data to identify. The digital image processing techniques is working currently in the research area to optimize the results, we have optimized the results. Our method shows better result as compare to the previous method in all cases such as error rate, psnr, mse, accuracy and ssim. In the future work, the face recognition system will provide the quality of live faces. There are limited issues that have to need to improve of the recognized faces. We have to need to optimize all the basic results in the face recognition system. The accuracy and quality are the biggest issues that can be improve using other new technique and may be optimize the live faces for the security purpose.

\section{REFERENCES}

[1] G. Aneesh M U, Abhishek A K Masand and K Manikantana, "Optimal Feature Selection based on Image Preprocessing using Accelerated Binary Particle Swarm Optimization for Enhanced Face Recognition", ELSEVIER, 750-758, 2011.

[2] Matthew A. Turk and Alex and P.Pentland, "Face Recognition Using Eigenfaces”, IEEE, 586-591, 1991.

[3] Priya Sisodia, Akhilesh Verma and Sachin Kansal, "Human Facial Expression Recognition using Gabor Filter Bank with Minimum Number of Feature Vectors ", International Journal of Applied Information Systems (IJAIS), 9-13, July 2013.

[4] Zhenhua Chai, Heydi Mendez-Vazquez, Ran He, Zhenan and Tienie Tan, "Explore semantic pixel sets based local patterns with information entropy for face recognition", Springer, 2014.

[5] J. Wang and Y. Tan, "Hausdorff distance with k-nearest neighbors," in Advances in Swarm Intelligence. Berlin, Germany: Springer-Verlag, pp. 272-281, 2012.

[6] Chandrappa D N and Ravishankar M "Gabor Wavelets And Morphological Shared Weighted Neural Network Based Automatic Face Recognition", Signal \& Image Processing: An International Journal (SIPIJ), Vol.4, No.4, 61-70, August 2013.

[7] Muhammad SHARIF, Adeeel KHALID, Mudassar RAZA and Sajjad MOHSIN, "Face Recognition using Gabor Filters", Journal of Applied Computer Science \& Mathematics, 53-55 , 2011.

[8] Dong Hui and Han Dian Yuan, "Research of image Matching Algorithm Based on SURF features", International Conference on Computer Science and Information Processing (ICCSIP), 2012.

[9] Shih-Wei Lin and Shih-Chieh Chen, "PSOLDA: A particle swarm optimization approach for enhancing classification accuracy rate of linear discriminated analysis", Elsevier, 1008-1015, 2009.

[10] David Bariena "Gabor wavelets in image processing".

[11] Gheorghita Ghinea, Rajkumar, Kannan, and Suresh Kannaiyan, "Gradient-Orientation-Based PCA Subspace for Novel Face Recognition”, IEEE, 914-920, August 2014.

[12] Ms. B. Saranya Bargavi and Ms C. Santhi, "Global and Local Facial Feature Extraction using Gabor Filters", International Journal of Science, Engineering and Technology Research (IJSETR), Volume 3, Issue 4, pp.10201023, April 2014

[13] Kennedy, J. a Dong Hui and Han Dian Yuan "Research of Image Matching Algorithm Based on SURF Features", International Conference on Computer Science and Information Processing (CSIP), 1140 - 1143, 2012.

[14] F. Bashar, A. Khan, F. Ahmed, and H. Kabir, "Face recognition using similarity pattern of image directional edge response," Advance Electronic Computer Engineering, vol. 14, no. 1, pp. 69-76, 2014.

[15] Lu, J, Plataniotis, K. N, and Venetsanopoulos and A. N, "Face recognition using LDA-based algorithms," IEEE Trans. on Neural Networks, 195-200, JANUARY 2003.

[16] M. A. Turk and A. P. Pentland, "Face recognition using eigenfaces," in Proceeding IEEE Computer Socciety Conf. Comput. Vis. Pattern Recognition, pp. 586591, Jun. 1991

[17] Ji-Sang Bae, Oh- young Lee and Jong-Ok Kim, "Image 
Interpolation Using Gabor Filter”, IEEE, 986-990, 2013.

[18] T. Archana, Dr, T. Venugopal and M. Praneeth Kumar, "Face Recognition Methodologies Using Component Analysis: The Contemporary Affirmation of the Recent Literature", Global Journal of Computer Science and Technology Graphics \& Vision (GJCSTGV), Volume 12, Issue 13, 2012.

[19] Sugreev Kaur and Rajesh Mehra, "High Speed And Area Efficient 2D DWT Processor Based Image Compression", SIPIJ, pp. 22-31, vol. 1, no.2, dec 2010.

\section{Authors' Profiles}

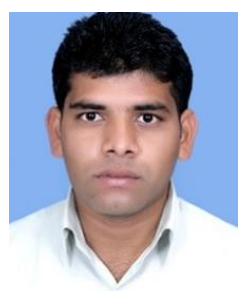

Narpat Singh has completed his four year bachelor degree of B.tech in computer Science and Engineering from Punjab Technical University, Jalandhar, Punjab, India in 2012. Currently, he is pursing two year master of M.Tech in computer Science and Engineering from Punjab Technical University, Jalandhar, Punjab, India. His specialization area is Digital Image Processing. He is certified with DB2, IBM RAD 6.0, IBM Web Sphere 6.2 and Awarded with IBM Blue Scholar. He is trying to research in Face Recognition for security purpose.

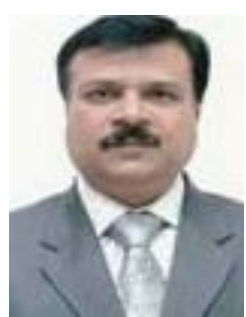

Manoj Kumar received his B.E (ECE) from Gulbarga University in the year 1990 and M.Tech. (ECE) from Punjab Technical University, Jalandhar in the year 2001. He completed his PhD from Punjab Technical University, Jalandhar in the year 2007. From 1991 to 2001, worked as lecturer and thereafter from July 2001 till July 2010 as Vice-Principal \& Faculty Head in Department of ECE and research centre at DAV Institute of Engineering \& Technology, Jalandhar. He joined CT Institute of Engineering Management \& Technology (CTIEMT), Jalandhar in July 2010 and presently working as the Group Director of CT Institutions, Jalandhar. He has published 62 research papers in the International/National Journals/Conferences, authored 08 Engineering books and reviewed 5 Engineering books. His area of interest is optical fiber communication, and wireless communication. He is life Member of ISTE and Punjab Academy of Sciences and fellow member IETE. He is reviewer for Elsevier Science's International Journal-Optical Fiber Technology, Springer, ICFAI Journals and World Scientific \& Engineering Academy and Society (WSEAS) for international conferences.

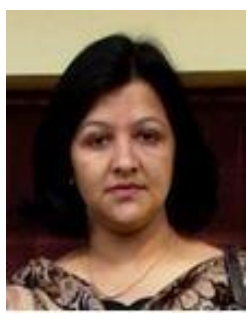

Manju Bala received her B.Tech. from U P Technical University Lucknow, India and Master of Technology in Computer Science and Engineering from Punjab Technical University Jalandhar in the year of 2007 and completed her Ph.d from NIT , Hamirpur (HP) in 2013 . She has worked eight years as a Lecturer in Information Technology at DAV Institute of Engineering and Technology, Jalandhar $(\mathrm{Pb})$.Currently she is working as Associate Professor and head of the Department in computer science and Engineering Departement at CT Insti- tute of Engineering Management and Technology, Jalandhar ( Punjab). Currently she is working in the area of data communication, computer network and wireless sensor networks. She has published 40 research papers in the International/National/Conferences. She is member of Punjab Science Congress, Patiala, India.

How to cite this paper: Narpat A. Singh, Manoj B. Kumar, Manju C. Bala, "Face Recognition System based on SURF and LDA Technique", International Journal of Intelligent Systems and Applications (IJISA), Vol.8, No.2, pp.13-19, 2016. DOI: 10.5815/ijisa.2016.02.02 